\title{
ERRORES EN LECTURA ORAL, VELOCIDAD LECTORA Y VELOCIDAD DE DENOMINACIÓN EN NIÑOS CON TDAH-DISLEXIA, DISLEXIA Y DESARROLLO NORMOLECTOR
}

\author{
Julián Palazón López \\ Coordinador del Grupo de Intervención en \\ Dificultades Específicas de Aprendizaje (INDEA) \\ julian.palazon@um.es
}

Recepción Artículo: 28 octubre 2020 Admisión Evaluación: 5 noviembre 2020 Informe Evaluador 1: 25 noviembre 2020

Informe Evaluador 2: 23 noviembre 2020 Aprobación Publicación: 27 noviembre 2020

\section{RESUMEN}

El Trastorno por Déficit de Atención e Hiperactividad (TDAH) y la dislexia son dos trastornos del neurodesarrollo frecuentemente comórbidos. Dos modelos han tratado de explicar este hecho. El modelo de fenocopia ha abogado porque TDAH y dislexia tienen bases neuronales y déficits cognitivos diferenciados, por lo que la intervención psicoeducativa relativa a sus dificultades en la lectura deberá estar diferenciada para ambos trastornos. En este modelo, las dificultades lectoras de un niño con TDAH serían cualitativamente diferentes de aquellas que presenta un niño con dislexia. Por contraposición, el modelo etiológico apuesta por bases neuronales y déficits cognitivos compartidos, donde la intervención psicoeducativa tendrá muchos puntos en común. Este modelo apuesta por que las dificultades en la lectura de un niño con TDAH son las que presentan los niños con dislexia. Desde esta encrucijada, consideramos que el análisis de errores en lectura oral, así como el estudio de variables relacionadas con la lectura (velocidad de denominación) podían resultar útiles para el refuerzo de uno de estos modelos. Por ello, se diseñó una investigación que nos permitiera indicar si dichas variables se distribuían de igual forma entre los tres grupos que componían los participantes del estudio (25 niños con TDAH-dislexia, 25 niños disléxicos y 25 niños normo lectores escolarizados en la Región de Murcia). El análisis estadístico llevado a cabo mediante la comparación de los errores y la velocidad de denominación de ambos grupos describió dificultades en la lectura similares entre el grupo TDAH-dislexia y el grupo dislexia, separándose ambos de manera semejante del grupo normo lector en las variables estudiadas, e infiriéndose afectados procesos cognitivos similares. Esto parece coincidir plenamente con el modelo etiológico, implicando que el abordaje de las dificultades en la lectura para niños con TDAH-dislexia estará basado en aquellas intervenciones que se hayan probado eficaces en la dislexia.

Palabras clave: dislexia; lectura oral; dificultad de aprendizaje; educación especial; investigación sobre la lectura 


\title{
ERRORES EN LECTURA ORAL, VELOCIDAD LECTORA Y VELOCIDAD DE DENOMINACIÓN EN NIÑOS CON TDAH-DISLEXIA, DISLEXIA Y DESARROLLO NORMOLECTOR
}

\begin{abstract}
Errors in oral reading, reading speed and speed of denomination in children with tdah-dislexia, dyslexia and normolector developmentattention. Deficit Hyperactivity Disorder (ADHD) and dyslexia are frequently comorbid. Two models have tried to explain this fact. The phenocopy model has defended that $\mathrm{ADHD}$ and dyslexia have neuronal bases and cognitive deficits that are different in both cases. In this case, the psychopedagogical intervention will be different for each disorder. From this model, ADHD children presents reading difficulties that are different those dyslexic children presents. By contrast, the etiological model defends shared neurological bases and cognitive deficits where the psychopedagogical intervention will have many points in common. This model understand that ADHD difficulties in are similar that dyslexic children presents. In this case, children presents simultaneously both disabilities. From this crossroads, we considerate that the qualitative analysis of oral reading mistakes and the study of other reading predictors (naming speed) could be useful to strengthen one of the models. Therefore, we designed a research to discover if these variables were distributed equally among the three groups that made up the study (25 children with ADHD-dyslexia, 25 dyslexic children and 25 children without reading problems). The statistical analysis carried out by comparing the errors and naming speed of both groups described similar reading difficulties between the ADHD-dyslexia group and the dyslexia group, both of them being separated in a similar way from the normal reader's group in the variables studied. It seems to coincide fully with the etiological model, implying a similar psychopedagocical intervention for children with ADHD and dyslexia that present reading disabilities.
\end{abstract}

Keywords: dislexia; oral Reading; learning diffculties; special education; reading research

\section{INTRODUCCIÓN}

En nuestro sistema educativo, las dificultades en la lectura son un grave problema, en tanto que impiden el acceso de nuestros alumnos a una habilidad instrumental básica para el desarrollo del individuo. Tanto el Trastorno por Déficit de Atención e Hiperactividad (TDAH) como la dislexia son trastornos frecuentemente comórbidos. Según Willcutt y Pennington (2000) entre el 15\% y el 35\% de los individuos que presentan dislexia cumplen también los criterios para ser diagnosticados como TDAH. La literatura científica ha venido entendiendo esta comorbilidad desde dos modelos opuestos (Jiménez y Díaz, 2014; Rosende-Vázquez, 2015). El primero de los modelos, denominado modelo de doble disociación o modelo de fenocopia, reconoce la comorbilidad entre los dos trastornos, aunque la considera un caso de fenocopia, donde, aunque los indicadores conductuales a menudo coincidan, las bases neurales y cognitivas afectadas para niños con TDAH y niños con dislexia son distintas. En este modelo, un trastorno A (TDAH o Dislexia) provocaría conductas propias de un trastorno B (Dislexia o TDAH, respectivamente), pero los dos trastornos no se darín a la vez (Jiménez y Díaz, 2014). En estos casos, parece lo más lógico que la intervención psicoeducativa trate de abordar, preferentemente, aquel trastorno que, de manera artificial, provoca las conductas propias del otro. El segundo modelo, denominado modelo de déficit múltiple, o modelo etiológico, establece que la alta comorbilidad viene dada por el hecho de que ambos trastornos comparten factores cognitivos y bases neurales, por lo tanto, la intervención educativa debería tener muchos puntos en común. En este caso los trastornos A y B (TDAH y dislexia) no están unidos por una relación de causalidad, sino que ambos se presentan a la vez, debido a que comparten déficits cognitivos (Jiménez y Díaz, 2014; Rosende-Vázquez, 2015) y genes (Willcutt et. al., 2002). La intervención psicoeducativa, en este caso, debe de hacer frente a ambos trastornos, entendiendo que los dos se dan simultáneamente. Con este trabajo se pretende comprobar en qué medida se ajustan ambos modelos a la realidad estudiada.

Para ello, se ha empleado el principio fundamental de que los errores en la lectura no son aleatorios, sino que nos permiten indagar en los défcits en el procesamiento cognitivo de aquellos sujetos que los cometen (Ramos y Cuetos, 1999). De ahí la suposición principal de este trabajo respecto al hecho de que la distribución de errores en lectura oral entre niños con TDAH-dislexia y niños con dislexia debe coincidir, en mayor o menor medida, con los modelos anteriormente explicados (distribución de errores desigual para ambas condiciones en el modelo de fenocopia; distribución similar de errores para ambas condiciones en el modelo etiológico). Además de la precisión en 
la lectura (errores en lectura oral) se evaluará la velocidad lectora, ya que esta habilidad se ha descrito como fundamental para una buena comprensión lectora (Álvarez-Cañizo, Suárez-Coalla y Cuetos, 2015).

La otra variable que complementa este trabajo, la velocidad de denominación, se ha reportado como una habilidad predictora de las habilidades lectoras (Aguilar et al., 2010; Suárez-Coalla, García-de-Castro y Cuetos, 2013) y como un indicador de dislexia (Wolf y Bowers, 1999). Una distribución igual o desigual de esta variable para ambos trastornos, coincidirá en mayor o menor medida con lo expuesto por ambos modelos.

Todo lo anterior se aborda desde el supuesto general de que, solo mediante la profunda comprensión de la realidad, podremos abordar la intervención psicoeducativa de la mejor manera posible.

\section{LOS ERRORES EN LECTURA ORAL: INDICADORES PARA EL DIAGNÓSTICO}

La clasificación de errores en lectura oral aportada por De las Heras (2002) nos sirvió para orientar nuestro trabajo. Estos son los errores, que van a evaluarse:

- Confusión: cuando se confunde el sonido de una letra o sílaba.

- Rotación: cuando se confunde el sonido de una letra por otra de igual forma pero diferente orientación espacial.

- Omisión: cuando se omite una letra, sílaba o palabra.

- Adición: cuando se agrega una letra, sílaba o palabra.

- Inversión: cuando se invierte la secuencia de las letras dentro de la palabra, o las palabras dentro de la frase.

- Sustitución: cuando se intercambia una palabra por otra.

- Contaminación: cuando no se entiende lo expresado por el niño.

- Disociación: cuando se segmenta una palabra en sus diferentes partes, existiendo una separación injustificada entre ellas.

\section{LA VELOCIDAD DE DENOMINACIÓN}

González, López, Villar y Rodríguez (2013) contemplan la velocidad de denominación como la capacidad de nombrar lo más rápidamente posible estímulos visuales altamente familiares, ya sean estos dígitos, letras, colores u objetos. Se trata de un predictor de la lectura que ha sido reconocido por numerosos investigadores en idiomas regulares como el español (Aguilar et al, 2010; Suárez-Coalla et al., 2013) Entendemos por idiomas regulares aqueIlos en los que cada grafema está relacionado con un número muy reducido de fonemas, por lo que su lectura y aprendizaje es más sencillo (Cuetos, González y De Vega, 2015).

En lo relativo a la relación de la velocidad de denominación con las dificultades en la lectura, el consenso no es absoluto (Jiménez y Hernández-Valle, 2014). Para algunos autores estaría relacionada con la velocidad de procesamiento (Guzmán et al., 2004; Wolf y Bowers, 1999), mientras que, para otros, es la manera de determinar la rapidez con la que puede recuperarse una determinada información fonológica almacenada en la memoria a largo plazo (Wagner, Torgesen y Rashote, 1994).

En la raíz de esta discusión se encuentra el trabajo de Wolf y Bowers (1999), quienes desarrollaron la conocida hipótesis de doble déficit, la cual postula que los niños con dislexia tienen afectadas la velocidad de denominación y el procesamiento fonológico, existiendo diferentes subtipos de dislexia en función de que el niño tenga afectadas una, 0 las dos variables.

En definitiva, la velocidad de denominación resulta interesante en nuestro estudio por dos motivos:

- Por la relevante relación que los estudios indican entre la velocidad de denominación y la lectura (Araujo, Reis, Petersson y Faísca, 2015).

- Por su relación con la velocidad de procesamiento, que ha sido indicada como deficitaria en niños con dislexia, y también en niños con TDAH (Shanahan et al., 2006).

\section{DISLEXIA}

Estudios sitúan la prevalencia de la dislexia en español en torno al 3\% de la población (Jiménez, Guzmán, Rodríguez y Artiles, 2009). 


\section{ERRORES EN LECTURA ORAL, VELOCIDAD LECTORA Y VELOCIDAD DE DENOMINACIÓN EN NIÑOS CON TDAH-DISLEXIA, DISLEXIA Y DESARROLLO NORMOLECTOR}

EI DSM-5 (Asociación Americana de Psiquiatría, 2013), pese a reconocer el término dislexia, lo engloba dentro de los trastornos del aprendizaje, definiéndolo como un patrón de dificultades del aprendizaje que se caracteriza por problemas con el reconocimiento de palabras, mal deletreo y poca capacidad ortográfica.

En la actualidad, pese a que el debate aun es intenso debido a los numerosos déficits reportados, y a que la dislexia sigue siendo aún un puzzle sin resolver (Cuetos, 2009) parece haber un amplio consenso en que la dislexia tiene, como uno de sus desencadenantes centrales, un déficit en el procesamiento fonológico (Alegría, 2006; Artigas-Pallarés, 2009; Dandache, Wouters \& Ghesquière, 2014; Defior y Serrano, 2011; Swank, 1999).

\section{TDAH}

El trastorno por déficit de atención e hiperactividad es, hoy, una realidad en nuestras aulas. Pese a que no conocemos estudios de prevalencia en nuestro país, estudios relevantes indican que la misma oscila alrededor del $5 \%$ en niños y adolescentes (Polanczyk \& Rohde, 2007). El DSM-5 (APA, 2013) define el TDAH como un patrón persistente de funcionamiento que se caracteriza por la falta de atención, la hiperactividad y la impulsividad. El inicio de algunos de los síntomas debe ser anterior a los 7 años y como mínimo uno de los síntomas debe presentarse en dos situaciones diferentes.

\section{Dos maneras de entender la comorbilidad TDAH-dislexia}

El modelo de doble disociación o modelo de fenocopia

Tal y como se ha introducido, según este modelo, TDAH y dislexia serían trastornos frecuentemente comórbidos, pero asociados a genes y a bases cognitivas distintas. (Jiménez y Díaz, 2014; Rosende-Vázquez, 2015). Serían un ejemplo de fenocopia, es decir, dos grupos de individuos que, sin tener el mismo genotipo, tienen en mismo fenotipo. Siguiendo esta lógica, llegado el momento de leer, niños con TDAH y niños disléxicos tendrían dificultades, pero cometerían errores diferentes A esto debemos añadir que este modelo explica la coexistencia del TDAH y la dislexia de la siguiente forma: el TDAH promueve las distracciones en el aula, dificulta el aprendizaje y, por tanto, deriva en un mayor número de errores y dificultades en la lectura. La dislexia, por su parte, hace que el niño esté desconectado y no preste atención a lo que sucede en el aula, ya que en la mayoría de los aprendizajes media la lectura, dando una sensación de inatención (Jiménez y Díaz, 2014; Rosende-Vázquez, 2015). Entonces, teniendo en cuenta estas afirmaciones y de acuerdo con este modelo, podríamos establecer los siguientes supuestos:

- Supuesto 1: Ios niños con TDAH y los niños con dislexia cometerán errores diferentes en lectura oral, al estar afectados procesos cognitivos diferentes.

- Supuesto 2: Ios niños con TDAH y los niños con dislexia responderán de manera diferente a la prueba de velocidad de denominación, por el motivo expresado en el supuesto anterior.

\section{El modelo de déficit múltiple o modelo etiológico}

Este modelo, en contraposición al anterior, defiende que TDAH y dislexia compartirían déficits cognitivos básicos que se derivarían de bases neurales que comparten muchas semejanzas (Artigas-Pallarés, 2009; Jiménez y Díaz, 2014; Rosende-Vázquez, 2015). Lo anterior solo parece plausible ante la eventualidad de que ambos trastornos compartan genes. Esto explicaría la alta comorbilidad, y además explicaría el carácter hereditario de ambos trastornos (Pennington, 2006).

Ante este hecho, cobran fuerza investigaciones como, por ejemplo, las de Willcutt et. al (2002), que determinan en el cromosoma $6 p$ una posible relación entre el TDAH y la dislexia.

Es posible formular los siguientes supuestos para este modelo:

- Supuesto 1: Ios niños con TDAH y los niños con dislexia cometerán errores similares en lectura oral, al estar afectados procesos cognitivos compartidos.

- Supuesto 2: Ios niños con TDAH y los niños con dislexia responderán de manera similar a la prueba de velocidad de denominación. , por el motivo expresado en el supuesto anterior. 


\section{METODOLOGÍA}

El presente artículo narra un estudio empírico de corte comparativo. Se considera que los estudios comparativos son aquellos que exploran la relación entre las variables, examinando las posibles diferencias entre grupos de individuos. Para ello se parte de las diferencias creadas entre dichos grupos por diferentes factores como la sociedad o la biología. No existe en estos estudios manipulación de la variable independiente, ni selección aleatoria de la muestra (Ato, López y Benavente, 2013).

A continuación, cabe presentar detalladamente las variables a analizar. En relación a las variables independientes, a partir de las cuáles se generarán los tres grupos donde se distribuirán los participantes de este trabajo, cabe distinguir entre:

- Diagnósticos de TDAH-dislexia

- Diagnósticos con trastornos de la lectura o dislexia.

- Niños normolectores 0 ausencia de diagnóstico.

\section{Participantes}

En este trabajo participaron un total de 75 niños escolarizados en centros educativos pertenecientes a la Región de Murcia, más concretamente en los cursos de cuarto y quinto de Educación Primaria. Dichos participantes se encuentran divididos en tres grupos:

- Un primer grupo, formado con 25 niños con TDAH con dificultades en la lectura

- Un segundo grupo, formado por 25 niños con dislexia

- Un tercer grupo, formado por 25 niños normolectores

En lo relativo a la selección de los participantes con TDAH y dislexia, se establecieron los siguientes criterios de inclusión:

- Que hubieran sido ya diagnosticados por los Equipos de Orientación y Educación Psicopedagógica de la Región de Murcia (EOEP) como niños con dificultades de aprendizaje compatibles con TDAH o dislexia.

- Que los niños pertenecieran a los cursos de cuarto y quinto de Educación Primara, ya que el diagnóstico suele sucerder a lo largo del tercer curso.

- En el caso de los niños ya diagnosticados con TDAH, bajo la orientación de los profesores se seleccionó a aquellos que presentaran también dificultades en la lectura.

Por su parte, los criterios de inclusión para la selección de los niños normolectores fueron los siguientes:

- Que los niños no hubieran sido identificados como alumnos con necesidades específicas de apoyo educativo (ACNEAE), siguiendo la denominación que indica la legislación actual (LOMCE, 2013).

- Que ocuparan un puesto aleatorio en el listado de clase, en concreto el número 10. En caso de que el mismo estuviera otorgado a un niño con necesidades específicas de apoyo educativo, se seleccionaría al niño que tuviera el número inmediatamente siguiente.

- Que estos niños pertenecieran a los mismos grupos de referencia que los niños TDAH y dislexia previamente seleccionados, con el propósito de reducir, en la medida de lo posible, las diferencias ambientales existentes relativas a los centros docentes.

A continuación (Tabla 1) se presenta la distribución de las variables edad y genero de los participantes de estudio, con la finalidad de aportar mayor información. 
Tabla 1. Distribución de las variables género, edad y curso para los participantes de este estudio.

\begin{tabular}{|c|c|c|c|}
\hline Grupo & Genero & Edad & Curso \\
\hline TDAH-dislexia & $\begin{array}{l}72 \% \text { Niños } \\
28 \% \text { Niñas }\end{array}$ & $\begin{array}{l}9 \text { años }(44 \%) \\
10 \text { años }(40 \%) \\
11 \text { años }(16 \%)\end{array}$ & $\begin{array}{l}60 \% 4^{\circ} \text { E.P. } \\
40 \% 5^{\circ} \text { E.P. }\end{array}$ \\
\hline Dislexia & $\begin{array}{l}\text { 64\%Niños } \\
36 \% \text { Niñas }\end{array}$ & $\begin{array}{l}9 \text { años }(24 \%) \\
10 \text { años }(36 \%) \\
11 \text { años }(24 \%)\end{array}$ & $\begin{array}{l}54 \% 4^{\circ} \text { E.P. } \\
46 \% 5^{\circ} \text { E.P. }\end{array}$ \\
\hline $\begin{array}{l}\text { Normo } \\
\text { Lector }\end{array}$ & $\begin{array}{l}46 \% \text { Niños } \\
54 \% \text { Niñas }\end{array}$ & $\begin{array}{l}9 \text { años }(48 \%) \\
10 \text { años }(40 \%) \\
11 \text { años }(12 \%)\end{array}$ & $\begin{array}{l}60 \% 4^{\circ} \text { E.P. } \\
40 \% 5^{\circ} \text { E.P. }\end{array}$ \\
\hline
\end{tabular}

\section{Instrumento}

Ninguno de los instrumentos que se emplearon para para la medición de las variables seleccionadas fue creado ad hoc para este trabajo. Para la presentación de dichos instrumentos conviene distinguir aquellos empleados para el análisis de los procesos de lectura oral de aquellos empleados para el análisis de la velocidad de denominación. En lo relativo al análisis de lectura oral, cabe destacar que fue necesario abordar dos requerimientos:

- La de recabar un material adecuado a la edad de los niños para que estos pudieran llevar a cabo una lectura oral adaptada a su nivel evolutivo.

- La de contar con un instrumento de evaluación adecuado para la valoración de dicha lectura oral.

El primero de los requerimientos se solventó empleando materiales pertenecientes a la Bateria de Evaluación de los Procesos Lectores PROLEC-R (Cuetos, Rodríguez, Ruano y Arribas, 2007) por dos motivos: en primer lugar, por haber sido diseñados estos materiales para la evaluación de niños de 6 a 12 años de edad, lo que abarcaba las edades comprendidas por los participantes de este trabajo; en segundo lugar, por contar esta prueba con materiales tanto destinados a los tres tipos de lectura que los niños realizaron:

- Lectura de texto

- Lectura de palabras

- Lectura de pseudopalabras

En lo relativo a la necesidad de contar con un instrumento para la evaluación de las producciones orales de los alumnos se usó el registro de análisis cualitativo de errores en lectural oral que el Equipo Específico de Dificultades de Aprendizaje de la Región de Murcia había diseñado anteriormente. Dicho registro se estimó conveniente ya que está basado en los mismos errores en lectura oral que De la Heras (2002) propone en su trabajo, y que se estimaron adecuados para la fundamentación de este estudio. Dicho instrumento fue supervisado por cuatro jueces que ejercían funciones docentes y/o de orientación educativa y son considerados expertos tanto en las dificultades en la lectura como en el aprendizaje de la misma.

En lo relativo a la velocidad de denominación, se empleó el test de velocidad de denominación de Fernández (2013), anteriormente citado. Dicho autor confirma en el manual la validez de dicha prueba, sosteniendo ésta en el hecho de que la prueba generaba diferencias significativas entre los grupos experimentales y el grupo control

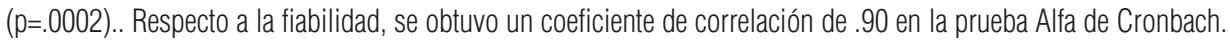

\section{RESULTADOS}

Debido a la necesidad de aplicar la estadística no paramétrica, se empleó la prueba U de Mann Whitney, con la intención de comprobar si existían diferencias significativas en los errores en la lectura oral, tanto entre los grupos TDAH-dislexia y dislexia, como entre cada uno de estos grupos por separado respecto al grupo normolector. 
En primer lugar, el análisis global (tras lectura de texto, palabras y psudopalabras) de los errores cometidos por el grupo TDAH-dislexia y normolector aportó resultados que indicaban la existencia de numerosas diferencias significativas entre ambos grupos (Tabla 2). De manera general, Ios niños con TDAH-dislexia tardaron más tiempo en realizar la lectura y cometieron más fallos, independientemente de la longitud de la palabra. De todos los errores categorizados, Ios niños TDAH-dislexia únicamente no mostraron diferencias significativas con el grupo normolector en los errores por inversión y contaminación.

Tabla 2. Análisis global de los errores en lectura. Prueba Mann-Whitney para grupos TDAH-dislexia y normolector.

\begin{tabular}{|c|c|c|c|c|}
\hline Aspecto analizado & $\begin{array}{c}\text { Grupos } \\
\text { analizados }\end{array}$ & $\begin{array}{l}\text { Rango } \\
\text { promedio }\end{array}$ & $\begin{array}{c}\text { Prueba U de } \\
\text { Mann- } \\
\text { Whitney }\end{array}$ & $\begin{array}{c}\text { Sig } \\
\text { asintótica } \\
\text { bilateral }\end{array}$ \\
\hline $\begin{array}{l}\text { Errores totales por } \\
\text { confusión en la lectura }\end{array}$ & $\begin{array}{l}\text { TDAH-dislexia } \\
\text { Normolector }\end{array}$ & $\begin{array}{l}32,24 \\
18,76\end{array}$ & 144,000 & ,001 \\
\hline $\begin{array}{l}\text { Errores totales por } \\
\text { rotación en la lectura }\end{array}$ & $\begin{array}{l}\text { TDAH-dislexia } \\
\text { Normolector }\end{array}$ & $\begin{array}{l}27,50 \\
23,50\end{array}$ & 262,500 & ,039 \\
\hline $\begin{array}{l}\text { Errores totales por } \\
\text { omisión de letras y } \\
\text { sílabas en la lectura }\end{array}$ & $\begin{array}{l}\text { TDAH-dislexia } \\
\text { Normolector }\end{array}$ & $\begin{array}{l}32,90 \\
18,10\end{array}$ & 127,500 &, 000 \\
\hline $\begin{array}{l}\text { Errores totales por } \\
\text { adición en la lectura }\end{array}$ & $\begin{array}{l}\text { TDAH-dislexia } \\
\text { Normolector }\end{array}$ & $\begin{array}{l}32,68 \\
18,32\end{array}$ & 133,000 &, 000 \\
\hline $\begin{array}{l}\text { Errores totales por } \\
\text { inversión en la lectura }\end{array}$ & $\begin{array}{l}\text { TDAH-dislexia } \\
\text { Normolector }\end{array}$ & $\begin{array}{l}28,56 \\
22,44\end{array}$ & 226,000 &, 117 \\
\hline $\begin{array}{c}\text { Errores totales por } \\
\text { contaminación en la } \\
\text { lectura }\end{array}$ & $\begin{array}{l}\text { TDAH-dislexia } \\
\text { Normolector }\end{array}$ & $\begin{array}{l}25,50 \\
25,50\end{array}$ & 312,500 & 1,000 \\
\hline $\begin{array}{l}\text { Errores totales por } \\
\text { sustitución en la lectura }\end{array}$ & $\begin{array}{l}\text { TDAH-dislexia } \\
\text { Normolector }\end{array}$ & $\begin{array}{l}31,48 \\
19,52\end{array}$ & 163,000 & ,003 \\
\hline $\begin{array}{l}\text { Errores totales por } \\
\text { disociación en la lectura }\end{array}$ & $\begin{array}{l}\text { TDAH-dislexia } \\
\text { Normolector }\end{array}$ & $\begin{array}{l}35,62 \\
15,38\end{array}$ & 59,500 &, 000 \\
\hline $\begin{array}{l}\text { Errores totales en la } \\
\text { lectura }\end{array}$ & $\begin{array}{l}\text { TDAH-dislexia } \\
\text { Normolector }\end{array}$ & $\begin{array}{l}35,54 \\
15,46\end{array}$ & 61,500 &, 000 \\
\hline $\begin{array}{c}\text { Errores totales en } \\
\text { palabras bisílabas en la } \\
\text { lectura }\end{array}$ & $\begin{array}{l}\text { TDAH-dislexia } \\
\text { Normolector }\end{array}$ & $\begin{array}{l}34,42 \\
16,58\end{array}$ & 89,500 &, 000 \\
\hline $\begin{array}{l}\text { Errores totales en } \\
\text { palabras de más de tres } \\
\text { sílabas en la lectura }\end{array}$ & $\begin{array}{l}\text { TDAH-dislexia } \\
\text { Normolector }\end{array}$ & $\begin{array}{l}36,12 \\
14,88\end{array}$ & 47,000 &, 000 \\
\hline $\begin{array}{l}\text { Tiempo de lectura total } \\
\text { en la lectura }\end{array}$ & $\begin{array}{l}\text { TDAH-dislexia } \\
\text { Normolector }\end{array}$ & $\begin{array}{l}35,32 \\
15,68\end{array}$ & 65,500 &, 000 \\
\hline
\end{tabular}




\section{ERRORES EN LECTURA ORAL, VELOCIDAD LECTORA Y VELOCIDAD DE DENOMINACIÓN EN NIÑOS CON TDAH-DISLEXIA, DISLEXIA Y DESARROLLO NORMOLECTOR}

Para la comparación de la globalidad de los errores cometidos en las diferentes lecturas entre el grupo dislexia y normolector, los análisis estadísticos mostraron diferencias significativas en, prácticamente, todos los aspectos analizados (véase Tabla 3). Los niños disléxicos tardaron más tiempo en la lectura y cometieron más errores, independientemente de la extensión de la palabra. Únicamente, no se encontraron diferencias significativas entre ambos grupos en los errores por contaminación.

Tabla 3. Análisis global de los errores en lectura. Prueba Mann-Whitney para grupos Dislexia y normolector

\begin{tabular}{|c|c|c|c|c|}
\hline Aspecto analizado & $\begin{array}{c}\text { Grupos } \\
\text { analizados }\end{array}$ & $\begin{array}{c}\text { Rango } \\
\text { promedio }\end{array}$ & $\begin{array}{c}\text { Prueba U de } \\
\text { Mann- } \\
\text { Whitney }\end{array}$ & $\begin{array}{l}\text { Sig } \\
\text { asintótica } \\
\text { bilateral }\end{array}$ \\
\hline $\begin{array}{l}\text { Errores totales por } \\
\text { confusión en la lectura }\end{array}$ & $\begin{array}{c}\text { Dislexia } \\
\text { Normolector }\end{array}$ & $\begin{array}{l}33,56 \\
17,44\end{array}$ & 111,000 &, 000 \\
\hline $\begin{array}{l}\text { Errores totales por } \\
\text { rotación en la lectura }\end{array}$ & $\begin{array}{c}\text { Dislexia } \\
\text { Normolector }\end{array}$ & $\begin{array}{l}27,50 \\
23,50\end{array}$ & 262,500 & 039 \\
\hline $\begin{array}{l}\text { Errores totales por } \\
\text { omisión de letras y } \\
\text { sílabas en la lectura }\end{array}$ & $\begin{array}{c}\text { Dislexia } \\
\text { Normolector }\end{array}$ & $\begin{array}{l}27,50 \\
23,50\end{array}$ & 103,000 &, 000 \\
\hline $\begin{array}{l}\text { Errores totales por } \\
\text { adición en la lectura }\end{array}$ & $\begin{array}{l}\text { Dislexia } \\
\text { Normolector }\end{array}$ & $\begin{array}{l}33,96 \\
17,04\end{array}$ & 101,000 &, 000 \\
\hline $\begin{array}{l}\text { Errores totales por } \\
\text { inversión en la lectura }\end{array}$ & $\begin{array}{c}\text { Dislexia } \\
\text { Normolector }\end{array}$ & $\begin{array}{l}29,64 \\
21,36\end{array}$ & 209,000 &, 035 \\
\hline $\begin{array}{c}\text { Errores totales por } \\
\text { contaminación en la } \\
\text { lectura }\end{array}$ & $\begin{array}{c}\text { Dislexia } \\
\text { Normolector }\end{array}$ & $\begin{array}{l}33,64 \\
17,36\end{array}$ & 312,500 & 1,000 \\
\hline $\begin{array}{c}\text { Errores totales por } \\
\text { sustitución en la lectura }\end{array}$ & $\begin{array}{c}\text { Dislexia } \\
\text { Normolector }\end{array}$ & $\begin{array}{l}33,64 \\
17,36\end{array}$ & 109,000 &, 000 \\
\hline $\begin{array}{c}\text { Errores totales por } \\
\text { disociación en la lectura }\end{array}$ & $\begin{array}{c}\text { Dislexia } \\
\text { Normolector }\end{array}$ & $\begin{array}{l}36,68 \\
14,32\end{array}$ & 33,000 &, 000 \\
\hline $\begin{array}{c}\text { Errores totales en la } \\
\text { lectura }\end{array}$ & $\begin{array}{c}\text { Dislexia } \\
\text { Normolector }\end{array}$ & $\begin{array}{l}37,14 \\
13,86\end{array}$ & 21,500 &, 000 \\
\hline $\begin{array}{c}\text { Errores totales en } \\
\text { palabras bisílabas en la } \\
\text { lectura }\end{array}$ & $\begin{array}{c}\text { Dislexia } \\
\text { Normolector }\end{array}$ & $\begin{array}{l}36,02 \\
14,98\end{array}$ & 49,500 &, 000 \\
\hline $\begin{array}{l}\text { Errores totales en } \\
\text { palabras de más de tres } \\
\text { sílabas en la lectura }\end{array}$ & $\begin{array}{c}\text { Dislexia } \\
\text { Normolector }\end{array}$ & $\begin{array}{l}37,52 \\
13,48\end{array}$ & 12,000 &, 000 \\
\hline $\begin{array}{l}\text { Tiempo de lectura total } \\
\text { en la lectura }\end{array}$ & $\begin{array}{c}\text { Dislexia } \\
\text { Normolector }\end{array}$ & $\begin{array}{l}36,34 \\
14,66\end{array}$ & 41,500 &, 000 \\
\hline
\end{tabular}

Para completar el análisis de errores en lectura oral, se aplicó el análisis estadístico a la globalidad de los errores cometidos entre el grupo TDAH-dislexia y el grupo dislexia (véase Tabla 4). Los resultados aportaron una total falta de diferencias significativas entre ambos grupos, en todos y cada uno de los aspectos valorados en este trabajo. 
Tabla 4. Análisis global de los errores en lectura. Prueba Mann-Whitney para grupos TDAH y dislexia.

\begin{tabular}{|c|c|c|c|c|}
\hline Aspecto analizado & $\begin{array}{c}\text { Grupos } \\
\text { analizados }\end{array}$ & $\begin{array}{l}\text { Rango } \\
\text { promedio }\end{array}$ & $\begin{array}{l}\text { Prueba U de } \\
\text { Mann- } \\
\text { Whitney }\end{array}$ & $\begin{array}{c}\text { Sig } \\
\text { asintótica } \\
\text { bilateral }\end{array}$ \\
\hline $\begin{array}{l}\text { Errores totales por } \\
\text { confusión en la lectura }\end{array}$ & $\begin{array}{l}\text { TDAH- } \\
\text { dislexia } \\
\text { Dislexia }\end{array}$ & $\begin{array}{l}24,36 \\
26,64\end{array}$ & 284,000 &, 577 \\
\hline $\begin{array}{l}\text { Errores totales por } \\
\text { rotación en la lectura }\end{array}$ & $\begin{array}{l}\text { TDAH- } \\
\text { dislexia } \\
\text { Dislexia }\end{array}$ & $\begin{array}{l}25,42 \\
25,58\end{array}$ & 310,500 & ,951 \\
\hline $\begin{array}{l}\text { Errores totales por } \\
\text { omisión de letras y } \\
\text { sílabas en la lectura }\end{array}$ & $\begin{array}{l}\text { TDAH- } \\
\text { dislexia } \\
\text { Dislexia }\end{array}$ & $\begin{array}{l}25,28 \\
25,72\end{array}$ & 307,000 & ,914 \\
\hline $\begin{array}{l}\text { Errores totales por } \\
\text { adición en la lectura }\end{array}$ & $\begin{array}{l}\text { TDAH- } \\
\text { dislexia } \\
\text { Dislexia }\end{array}$ & $\begin{array}{l}23,72 \\
27,28\end{array}$ & 268,000 & ,381 \\
\hline $\begin{array}{l}\text { Errores totales por } \\
\text { inversión en la lectura }\end{array}$ & $\begin{array}{l}\text { TDAH- } \\
\text { dislexia } \\
\text { Dislexia }\end{array}$ & $\begin{array}{l}24,18 \\
26,82\end{array}$ & 279,500 & ,506 \\
\hline $\begin{array}{c}\text { Errores totales por } \\
\text { contaminación en la } \\
\text { lectura }\end{array}$ & $\begin{array}{l}\text { TDAH- } \\
\text { dislexia } \\
\text { Dislexia }\end{array}$ & $\begin{array}{l}25,50 \\
25,50\end{array}$ & 312,500 & 1,000 \\
\hline $\begin{array}{c}\text { Errores totales por } \\
\text { sustitución en la lectura }\end{array}$ & $\begin{array}{l}\text { TDAH- } \\
\text { dislexia } \\
\text { Dislexia }\end{array}$ & $\begin{array}{l}22,76 \\
28,24\end{array}$ & 244,000 &, 180 \\
\hline $\begin{array}{l}\text { Errores totales por } \\
\text { disociación en la lectura }\end{array}$ & $\begin{array}{l}\text { TDAH- } \\
\text { dislexia } \\
\text { Dislexia }\end{array}$ & $\begin{array}{l}23,98 \\
27,02\end{array}$ & 274,500 & ,461 \\
\hline $\begin{array}{l}\text { Errores totales en la } \\
\text { lectura }\end{array}$ & $\begin{array}{l}\text { TDAH- } \\
\text { dislexia } \\
\text { Dislexia }\end{array}$ & $\begin{array}{l}23,82 \\
27,18\end{array}$ & 270,500 & ,415 \\
\hline $\begin{array}{c}\text { Errores totales en } \\
\text { palabras bisílabas en la } \\
\text { lectura }\end{array}$ & $\begin{array}{l}\text { TDAH- } \\
\text { dislexia } \\
\text { Dislexia }\end{array}$ & $\begin{array}{l}23,38 \\
27,62\end{array}$ & 259,500 & ,303 \\
\hline $\begin{array}{l}\text { Errores totales en } \\
\text { palabras de más de tres } \\
\text { sílabas en la lectura }\end{array}$ & $\begin{array}{l}\text { TDAH- } \\
\text { dislexia } \\
\text { Dislexia }\end{array}$ & $\begin{array}{l}22,76 \\
28,24\end{array}$ & 244,000 & ,183 \\
\hline $\begin{array}{l}\text { Tiempo de lectura total } \\
\text { en la lectura }\end{array}$ & $\begin{array}{l}\text { TDAH- } \\
\text { dislexia } \\
\text { Dislexia }\end{array}$ & $\begin{array}{l}24,86 \\
26,14\end{array}$ & 296,500 & ,756 \\
\hline
\end{tabular}


Por último, en lo relativo a la velocidad de denominación, los resultados (véase Tabla 5) indican que grupos TDAH-dislexia y dislexia denominaron imágenes con un tiempo de latencia significativamente mayor que el grupo normolector. Sin embargo, no hubo diferencias significativas en la velocidad de denominación para ambos grupos.

\begin{tabular}{|c|c|c|c|c|}
\hline Aspecto analizado & $\begin{array}{l}\text { Grupos } \\
\text { analizados }\end{array}$ & $\begin{array}{c}\text { Rango } \\
\text { promedio }\end{array}$ & $\begin{array}{c}\text { Prueba U de } \\
\text { Mann- } \\
\text { Whitney }\end{array}$ & $\begin{array}{c}\text { Sig } \\
\text { asintótica } \\
\text { bilateral }\end{array}$ \\
\hline $\begin{array}{l}\text { Tiempo empleado en el } \\
\text { Test de Velocidad de } \\
\text { Denominación }\end{array}$ & $\begin{array}{c}\text { TDAH } \\
\text { Normolector }\end{array}$ & $\begin{array}{l}31,26 \\
19,74\end{array}$ & 168,500 &, 005 \\
\hline $\begin{array}{l}\text { Tiempo empleado en el } \\
\text { Test de Velocidad de } \\
\text { Denominación }\end{array}$ & $\begin{array}{c}\text { Dislexia } \\
\text { Normolector }\end{array}$ & $\begin{array}{l}33,00 \\
18,00\end{array}$ & 125,000 &, 000 \\
\hline $\begin{array}{l}\text { Tiempo empleado en el } \\
\text { Test de Velocidad de } \\
\text { Denominación }\end{array}$ & $\begin{array}{c}\text { TDAH } \\
\text { Dislexia }\end{array}$ & $\begin{array}{l}25,00 \\
26,00\end{array}$ & 300,000 &, 808 \\
\hline
\end{tabular}

\section{DISCUSIÓN}

La interpretación primera, y más general, que se deriva del análisis de los resultados es que tanto los niños con TDAH-dislexia como los niños disléxicos realizaron las lecturas que se les presentaron con más dificultad, tardando más y cometiendo más errores que los niños normolectores. Pero ¿están estas dificultades relacionadas entre sí?

La respuesta a este interrogante, en lo que a esta investigación se refiere, deja lugar a pocas dudas. Destaca aquí el hecho de que tanto el grupo TDAH-dislexia como el grupo dislexia no solo comparte un mayor número de errores, sino que, además, dichos errores, son prácticamente similares y las variables presentadas para su estudio se distribuyen sin que existan diferencias significativas entre ambos grupos. Esto parece coincidir con lo expuesto por aquellos autores que afirman que TDAH y dislexia poseen déficits cognitivos compartidos (Artigas-Pallarés, 2009; Rosende-Vázquez, 2015), que parten de disfunciones neurológicas comunes entre ambos grupos, y que podrían un origen genético compartido (Willcutt et. al, 2002).

Por todo lo anterior, los resultados obtenidos se explican mejor a través de lo expuesto por el modelo de déficit múltiple o modelo etiológico (Artigas-Pallarés, 2009; Jiménez y Díaz, 2014; Rosende-Vázquez, 2015).

No obstante, Ramos y Cuetos (1999) plantean la posibilidad de realizar, de manera inductiva, un análisis de los procesos cognitivos que están dificultando la lectura a partir del análisis de errores. Para ello, resulta interesante comenzar por analizar el error más cometido por los niños con TDAH-dislexia y dislexia, el error por disociación. Ambos grupos obtuvieron diferencias significativas en ambas variables respecto al grupo normolector, aunque, por el contrario, no mostraron diferencias significativas entre sí. Se considera error por disociación aquel que fragmenta una palabra de forma incorrecta, no consiguiendo leer la misma como un todo (De las Heras, 2002). Se puede apreciar, especialmente durante la lectura del texto, que se registraban gran número de errores en palabras largas como incendiaban, transportarlas o embarcaciones, donde los niños disociaban la palabra, separando sus partes, de manera que la palabra no llegaba a pronunciarse como un todo, por lo quedaba registrado el error.

Que ambos grupos tuvieran dificultades en las palabras largas, y que estos errores consistieran, mayoritariamente, en la incapacidad de decodificar mentalmente los símbolos con la suficiente rapidez para que la palabra se pronunciara como un todo nos Ileva, inmediatamente, a relacionar estos resultados con las dificultades en el 
procesamiento fonológico características de la dislexia y que indican un nulo reconocimiento automático de palabras (Defior y Serrano, 2011).

Estas dificultades se complementan, en este trabajo, con otros errores de carácter fonológico, a los que también se les asigna un papel central en la dislexia (Alegría, 2006; Defior y Serrano, 2011; Swank, 1999).

Los errores asignados a las dificultades en la ruta fonológica (Jiménez, 2014) tales como aquellos que se producen en la conversión de grafemas a fonemas concretos, con sonido o forma similar (errores por confusión), o los que se producen por lexicalización, reemplazando una palabra por otra que guarda cierta semejanza, al no poder decodificarla bien (errores por sustitución) se mostraron, igualmente, en mayor medida tanto en niños con TDAHdislexia como niños con dislexia. También muestran ambos grupos diferencias significativas respecto al grupo normolector en otros errores asociafonemas dos a la ruta fonológica, tales como aquellos en los que la ordenación de los que se produce de manera incorrecta (errores por inversión).

Para los problemas asociados a la ruta visual u ortográfica vuelve a reproducirse el mismo hecho. Grupos TDAH-dislexia y dislexia muestran dificultades significativas respecto al grupo normolector, sin embargo, no muestran diferencias significativas entre sí. Ambos grupos muestran cierta incapacidad para reconocer inmediatamente la imagen visual de una palabra, especialmente si esta es larga o poco frecuente. Estas dificultades para reconocer las palabras de forma directa obligan a los alumnos a decodificar todas las palabras por la ruta fonológica, mucho más lenta (Jiménez, 2014), Io que vendría a explicar el hecho de que los alumnos con TDAH-dislexia y los alumnos con dislexia tarden un tiempo significativamente mayor, en relación al grupo normolector, en todas las lecturas realizadas, siendo sus tiempos de lectura similares entre sí.

En definitiva, Ios resultados nos informan de que tanto el grupo dislexia como el grupo TDAH-dislexia cumplían con todos los supuestos que, tradicionalmente, se atribuyen a los niños con dislexia durante la lectura, siendo especialmente relevantes aquellos relacionados con el procesamiento fonológico (Alegría, 2006; Defior y Serrano, 2011; Swank, 1999).

Para reforzar los anteriores resultados, en lo relativo a la velocidad de denominación, tanto el grupo TDAH-dislexia como el grupo dislexia mostraron diferencias significativas respecto al grupo normolector. No obstante, no se encontraron diferencias significativas en la velocidad de denominación entre los niños con TDAH-dislexia y los niños disléxicos. Estos resultados concuerdan con los obtenidos con otros investigadores en trabajos anteriores que analizaban la velocidad de denominación entre niños con TDAH y niños con dislexia (Shanahan et al., 2006).

La velocidad de denominación se ha establecido como indicador de la dislexia (Wolf y Bowers, 1999) y como predictor de las dificultades en la lectura (Aguilar et. Al, 2010; Suárez-Coalla et al., 2013). Que ambas grupos compartan dicha denominación lenta de dibujos nos advierte, una vez más, de que las dificultades en la lectura para ambos trastornos no parecen devenir de una causalidad artificial.

\section{CONCLUSIONES}

Niños con dislexia y niños con TDAH-dislexia cometieron más errores en la lectura que el grupo normolector. Lo más relevante es que, para estos dos grupos, los errores no fueron diferentes ni en cantidad ni en forma.

Además, en lo relativo a la velocidad de denominación, volvió al darse la misma pauta. Niños con dislexia y niños con TDAH-dislexia denominaron las imágenes significativamente más despacio que el grupo normolector, no obstante, entre ambos grupos no se percibieron diferencias significativas.

Por todo lo anterior, los resultados de este estudio se ajustan, a nuestro juicio, de manera rotunda al modelo de déficit múltiple o modelo etiológico.

Los resultados de esta investigación, que a nuestro parecer se explican mejor a través de este modelo son:

- Que el análisis global de errores en lectura oral no exprese la existencia de diferencias significativas entre Ios grupos TDAH-dislexia y dislexia.

- Que el análisis global de errores en lectura oral indique que tanto el grupo TDAH-dislexia como el grupo dislexia muestran, exactamente, las mismas diferencias significativas respecto al grupo normolector. 


\section{ERRORES EN LECTURA ORAL, VELOCIDAD LECTORA Y VELOCIDAD DE DENOMINACIÓN EN NIÑOS CON TDAH-DISLEXIA, DISLEXIA Y DESARROLLO NORMOLECTOR}

- Que el empleo de las rutas ortográfica y fonológica se adecúe, por parte de los dos grupos, a lo que la literatura científica establece como habitual para las dificultades en la lectura de los niños disléxicos. Se deduce de los errores similares la existencia de déficits cognitivos compartidos (Ramos y Cuetos, 1999).

- Que la velocidad de denominación fuera igualmente deficitaria, en ambos grupos respecto al grupo normolector, siendo esta un predictor de la lectura y un contrastado indicador de disexia.

La consecuencia psicoeducativa más relevante que podría derivarse de este trabajo tiene que ver con la necesidad de establecer, también para los niños que presenten la comorbilidad TDAH-dislexia procesos de rehabilitación fonológica propios de la intervención en dislexia, acompañados de los tratamientos cognitivo-conductuales propios del TDAH (Pérez, 2015, Tamm et al., 2017).

Existe una amplia evidencia empírica que indica las ventajas de iniciar el tratamiento de la dislexia de manera temprana, esgrimiéndose razones relacionadas con la plasticidad neural (Papanicolau et al., 2003). Si descartáramos el hecho de que el Trastorno A (TDAH), pudiera generar conductas propias del Trastorno B (dislexia), sería fácil llegar a la conclusión de que aquellos niños con déficit de atención e hiperactividad que presenten dificultades en la lectura se beneficiarán de que sus problemas lectores se aborden lo antes posible con intervenciones centradas en el déficit fonológico propias de la dislexia (Galuschka, Ise, Krick \& Schulte-Körne, 2014).

\section{REFERENCIAS BIBLIOGRÁFICAS}

Aguilar, M., Navarro, J. I., Menacho, I., Alcalde, C., Marchena, E., y Ramiro, P. (2010). Velocidad de nombrar y conciencia fonológica en el aprendizaje inicial de la lectura. Psicothema, 22(3), 436-442

Alegría, J. (2006). Por un enfoque psicolingüístico de la lectura y sus dificultades 20 años después. Infancia y Aprendizaje, 29(1), 93-111.

Álvarez-Cañizo, M., Suárez-Coalla, P., \& Cuetos, F. (2015). The role of reading fluency in children's text comprehension. Frontiers in psychology, 6, 1810.

Araújo, S., Reis, A., Petersson, K. M., \& Faísca, L. (2015). Rapid automatized naming and reading performance: A meta-analysis. Journal of Educational Psychology, 107(3), 868-883.

Artigas-Pallarés, J. (2009). Dislexia: enfermedad, trastorno o algo distinto. Revista de neurología, 48(2), 63-69.

Ato, M., López, J. J., y Benavente, A. (2013). Un sistema de clasificación de los diseños de investigación en psicología. Anales de psicología, 29(3), 1038-1059.

Cuetos, F. (2009). Dislexias evolutivas: un puzzle por resolver. Revista de logopedia, foniatría y audiología, 29(2), 78-84.

Cuetos, F., González, J., \& De Vega, M. (2015). Psicología del lenguaje. Madrid, España: Ed. MédicaPanamericana.

Cuetos, F., Rodríguez, B., Ruano; E. y Arribas, D. (2007). Evaluación de los procesos lectores revisada PROLECR. Madrid, España: Tea.

Dandache, S., Wouters, J., \& Ghesquière, P. (2014). Development of reading and phonological skills of children at family risk for dyslexia: a longitudinal analysis from kindergarten to sixth grade. Dyslexia, 20(4), 305-329.

De Las Heras, J. (2002). Estudio de perfiles evolutivos en la lectura. Validación y revisión del test individual de diagnóstico de errores en lectura (TIDEL). Tesis doctoral. Valencia, España: Universidad de Valencia.

De Psiquiatría, A. A. (2013). Manual diagnóstico y estadístico de los trastornos mentales- DSM-5. Arlington, EE:UU: Médica Panamericana.

Defior, S., y Serrano, F. (2011). Procesos fonológicos explícitos e implícitos. Revista Neuropsicología, Neuropsiquiatría y Neurociencias, 11(1), 79-94.

Fernández, A. L. (2013). Development of a confrontation naming test for spanish speakers: The cordoba Naming Test. The clinical neuropshichologist, 27(7), 1179-1198 Córdoba: Universidad de Córdoba.

Galuschka, K., Ise, E., Krick, K., \& Schulte-Körne, G. (2014). Effectiveness of treatment approaches for children and adolescents with reading disabilities: a meta-analysis of randomized controlled trials. PloS one, 9(2), e89900. 
González, R.M., López, S., Villar, J. y Rodríguez, A. (2013). Estudio de los predictores de la lectura. Revista de Investigación en Educación, 11 (2), 98-110.

Guzmán, R., Jiménez, J. E., Ortiz, M. R., Hernández Valle, I., Estévez, A., Rodrigo, M. y Hernández, S. (2004). Evaluación de la velocidad de nombrar en las dificultades de aprendizaje de la lectura. Psicothema, 16(3).

Jiménez, J.E. y Díaz, A. (2014). Comorbilidad con otros trastornos del desarrollo: dislexia y trastorno por déficit de atención y/o hiperactividad. En J. E. Jiménez (Coord.), Dislexia en español. Madrid, España: Pirámide.

Jiménez, J. E., Guzmán, R., y Rodríguez, C. y Artiles, C. (2009). Prevalencia de las dificultades específicas de aprendizaje: la dislexia en español. Anales de psicología, 25(1), 78.

Jiménez, J. E. y Hernández-Valle, I. (2014). Indicadores cognitivos de la dislexia. En J. E. Jiménez (Coord.), Dislexia en español. Madrid, España: Pirámide.

Jiménez, J.E. (2014). ¿Qué es la dislexia?. En J. E. Jiménez (Coord.), Dislexia en español. Madrid, España: Pirámide.

Ley Orgánica para la Mejora de la Calidad Educativa (LOMCE) (Ley Orgánica 8/2013, 9 de diciembre). Boletín Oficial del Estado, no 295, 2013, 10 diciembre.

Papanicolaou, A. C., Simos, P. G., Breier, J. I., Fletcher, J. M., Foorman, B. R., Francis, D., ... \& Davis, R. N. (2003). Brain mechanisms for reading in children with and without dyslexia: A review of studies of normal development and plasticity. Developmental Neuropsychology, 24(2-3), 593-612.

Pennington, B.F. (2006). From single to multiple deficit models of developmental disorders. Cognition, 101, 385-413.

Pérez, M. R. (2015). Tratamiento cognitivo-conductual de conductas disruptivas en un niño con TDAH y trastorno negativista desafiante. Revista de Psicología Clínica con niños y adolescentes, 2(1), 45-54.

Polanczyk, G., \& Rohde, L. A. (2007). Epidemiology of attention-deficit/hyperactivity disorder across the lifespan. Current opinion in psychiatry, 20(4), 386-392.

Ramos, J.L., y Cuetos, F. (1999). PROLEC: Evaluación de los procesos lectores en alumno del tercer ciclo de educación primaria y educación secundaria obligatoria. Madrid, España: TEA Ediciones.

Rosende-Vázquez, M. (2015). Acceso al léxico y atención selectiva en sujetos con TDAH, dislexia y combinado. Tesis doctoral. A Coruña, España: Universidad da Coruña.

Shanahan, M.A., Pennington, B.F., Yerys, B.E., Scott, A., Boada, R., Willcutt, E.G., Olson,R.K. y DeFries, J.C. (2006). Processing Speed Deficits in Attention Deficit/Hyperactivity Disorder and Reading Disability. Journal of Abnormal Child Psychology , 34, 585-602

Swank, L. K. (1999). Specific developmental disorders. The language-learning continuum. Child and adolescent psychiatric clinics of North America, 8(1), 89-112.

Suárez-Coalla, P., García-de-Castro, M., y Cuetos, F. (2013). Variables predictoras de la lectura y la escritura en castellano. Infancia y aprendizaje, 36(1), 77-89.

Tamm, L., Denton, C. A., Epstein, J. N., Schatschneider, C., Taylor, H., Arnold, L. E., ... \& Maltinsky, J. (2017). Comparing treatments for children with ADHD and word reading difficulties: A randomized clinical trial. Journal of consulting and clinical psychology, 85(5), 434.

Wagner, R. K., Torgesen, J. K., \& Rashotte, C. A. (1994). Development of reading-related phonological processing abilities: New evidence of bidirectional causality from a latent variable longitudinal study. Developmental psychology, 30(1), 73.

Willcutt, E. G., \& Pennington, B. F. (2000). Comorbidity of reading disability and attention-deficit/hyperactivity disorder: Differences by gender and subtype. Journal of learning disabilities, 33(2), 179-191.

Willcutt, E. G., Pennington, B. F., Smith, S. D., Cardon, L. R., Gayán, J., Knopik, \& DeFries, J. C. (2002). Quantitative trait locus for reading disability on chromosome $6 p$ is pleiotropic for attention deficit/hyperactivity disorder. American Journal of Medical Genetics Part A, 114(3), 260-268.

Wolf, M., \& Bowers, P. G. (1999). The double-deficit hypothesis for the developmental dyslexias. Journal of educational psychology, 91(3), 415-438. 
